\title{
A FAULT DETECTION TECHNIQUE FOR VARIABLE-SPEED WIND TURBINES USING VIBRATIONS AND ELECTRICAL MEASUREMENTS
}

\author{
J. M. Bossio, G. R. Bossio, C. H. De Angelo \\ Grupo de Electrónica Aplicada, Fac. de Ingeniería, Universidad Nacional de Río Cuarto, \\ Ruta Nac. \#36 Km. 601, (X5804BYA) Río Cuarto, Córdoba, Argentina. \\ jbossio@ing.unrc.edu.ar
}

\begin{abstract}
A technique for detecting faults in variable speed wind turbines with permanent magnet synchronous generators is proposed. The proposal consist on sampling and process vibration signals at constant position intervals, in order to obtain a speed independent vibration spectrum. The angular position information is obtained using a reduced order observer which allows estimating rotor position using voltage and current measurements, without the need of a position sensor. The estimated position is used for resampling vibration signals at constant position intervals with a high number of samples per revolution. Experimental results including a rotor imbalance fault are presented to validate the proposal.
\end{abstract}

Keywords - Fault detection, Permanent magnet synchronous generator, Vibration, Wind turbines.

\section{INTRODUCTION}

The increased use of renewable energy, particularly wind power, has increased interest in the development of condition monitoring techniques for these equipments [1]-[8]. Fault detection can prevent significant losses due to both generator breakage or other element of the turbine as well as the costs associated with energy not generated while the equipment is repaired. In general, wind turbines are located in difficult accessed places and hence, the significant importance of the automatic fault detection strategies that quite aid to lower the maintenance costs [9].

Wind turbine faults can be originated in the impeller including blades imbalance, torque oscillations, flow problems, among other external disturbances, and those associated with the transmission or mechanical coupling problems, such as axes misalignment or faulty gears [10][12]. Faults in generators, on the other hand, can be electrical, mechanical, or a combination of both; or electrical faults whose origin was a mechanical or vice versa. Most internal machine faults occur in the stator and in the bearings, and, in a lesser degree, in the rotor [13],[14].

Recently, Permanent Magnet Synchronous Generators (PMSG) have been used in wind turbines due to their high power density. This allows constructing generators with a high number of poles, thereby avoiding the use of heavy gearboxes [15],[16].

Usually, vibration analysis is used for monitoring the condition of gearbox, bearings and blades, while the analysis

Manuscript received 03/02/2014; revised 28/04/2014; accepted for publication 11/11/2014, by recommendation of the Special Section Editor Mário Lúcio da Silva Martins.

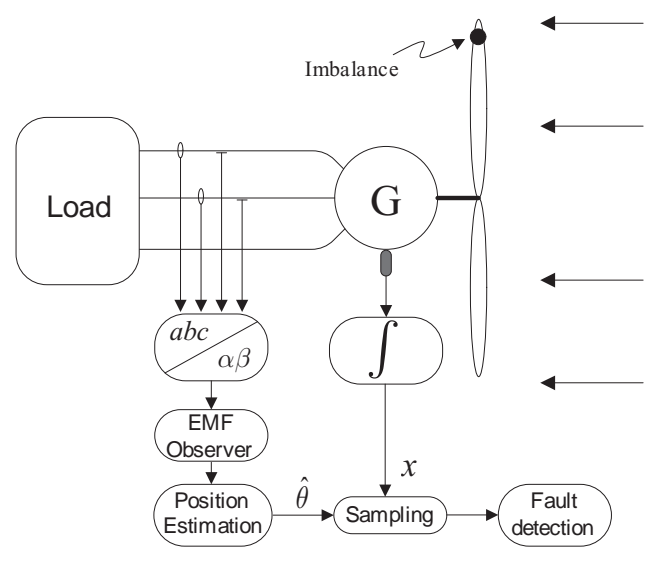

Fig. 1. General scheme of the proposed strategy.

of generator current spectrum is usually used for detecting faults in the generator itself.

For these reasons, condition monitoring systems in wind turbines usually include accelerometers (or another vibration sensors) placed in various points of the turbine. Besides, voltage and current sensors are included for both, protection or monitoring, and for control purposes [17],[18]. In any case, it is necessary to identify the operating frequencies and calculate the specific frequencies of faulty components. However, the main problem for condition monitoring of wind turbines is their operation at variable speed and load, which makes necessary to modify standard signal processing techniques to take into account such conditions [19].

A new method for processing vibration signals is proposed in this work. The proposed technique is based on a resampling of the acquired signals in order to obtain a speed independent vibration spectrum. Unlike previous works, such as [19], the resampling is obtained using a reduced order observer which allows estimating rotor position using generator voltage and current measurements, without the need of a position sensor. Moreover, due to high resolution in the position provided by the observer, a higher number of position samples per revolution is obtained, which makes the proposed resampling less sensitive to the interpolation method used.

\section{PROPOSED STRATEGY}

Figure 1 shows a general scheme of the proposed strategy. Based on the measurements of generator voltages and currents, a reduced order observer is designed in order to estimate the rotor position without using a position sensor. From 
the estimated position, vibration measurements are resampled at constant position intervals, in order to obtain a speedindependent signal used to detect and diagnose the faults.

\section{A. PMSG Model}

In order to develop the position estimation observer, the dynamic model of a PMSG is written in a stationary reference frame $\alpha \beta$ [20],

$$
\begin{aligned}
\frac{d i_{\alpha}}{d t} & =-\frac{R}{L} i_{\alpha}+\frac{1}{L} e_{\alpha}-\frac{1}{L} v_{\alpha} \\
\frac{d i_{\beta}}{d t} & =-\frac{R}{L} i_{\beta}+\frac{1}{L} e_{\beta}-\frac{1}{L} v_{\beta},
\end{aligned}
$$

$$
\begin{aligned}
& \frac{d \theta}{d t}=\omega \\
& \frac{d \omega}{d t}=-\frac{1}{J}\left(\varphi_{\alpha}(\theta) i_{\alpha}+\varphi_{\beta}(\theta) i_{\beta}\right)-\frac{B}{J} \omega-\frac{1}{J} T_{d},
\end{aligned}
$$

where $i_{\alpha}, i_{\beta}, e_{\alpha}, e_{\beta}, v_{\alpha}, v_{\beta}$ represent the current, electromotive force (EMF) and stator voltage components, respectively, in the stationary reference frame $\alpha \beta ; R$ and $L$ are the stator resistance and inductance, respectively; $\theta$ and $\omega$ represent the rotor position and speed; $J$ and $B$ are the inertia and viscosity, respectively; and $T_{d}$ is the wind turbine driving torque.

The EMF induced into the stator windings is given by,

$$
\begin{aligned}
& e_{\alpha}=\frac{\partial \lambda_{\alpha}}{\partial \theta} \frac{d \theta}{d t}=\varphi_{\alpha}(\theta) \omega \\
& e_{\beta}=\frac{\partial \lambda_{\beta}}{\partial \theta} \frac{d \theta}{d t}=\varphi_{\beta}(\theta) \omega,
\end{aligned}
$$

where $\lambda_{\alpha}$, and $\lambda_{\beta}$ are the linked flux components, and $\varphi_{\alpha}$, and $\varphi_{\beta}$ are the components of the flux derivative with respect to the rotor position.

In a sinusoidal PMSG, $\varphi_{\alpha}$ and $\varphi_{\beta}$ are sinusoidal functions of the position whereas in trapezoidal PMSGs they are trapezoidal. However, in many PMSG, these functions are neither sinusoidal nor trapezoidal [21]. For this reason $\varphi_{\alpha}$ and $\varphi_{\beta}$ are functions to be determined according to the generator type. These functions can be previously determined in an experimental way by measuring the instantaneous voltage, rotor position and speed under no-load condition. Once determined, these waveforms can be incorporated into the observer model as proposed in [22].

For simplicity, a sinusoidal PMSG is considered in this work, which is a particular case of the more general one presented in [22]. Therefore, for a sinusoidal PMSG is enough to consider only the fundamental component of the EMF waveforms. In this case, $\alpha \beta$ components of the position derivatives of flux are give by,

$$
\begin{aligned}
& \varphi_{\alpha}(\theta)=-\Phi \sin (\theta) \\
& \varphi_{\beta}(\theta)=\Phi \cos (\theta),
\end{aligned}
$$

where $\Phi$ is the magnitude of the rotor flux.

\section{B. Nonlinear Observer}

The information of speed and rotor position is contained in the induced EMF, as shown in (3). Therefore, it could be possible to estimate the induced EMF by measuring currents and voltages, and then calculate the rotor position from the estimated EMF. Position estimation from induced EMF may be inaccurate or unfeasible at low or zero speed [23]. However, since at low wind speed the energy that can be extracted from the wind is very low, wind turbines always operate over a minimum speed (cut-in speed) [24]-[26]. In variable speed wind turbines with PMSG, the cut-in speed is about 0.25 and $0.4 \mathrm{pu}$ [27]-[28]. Thus, EMF based rotor position estimation is a good option for the operating range of such wind turbines.

In order to estimate the rotor position, the time derivative of EMF can be calculated from (3) and taking into account (4),

$$
\begin{aligned}
\frac{d e_{\alpha}}{d t} & =-\varphi_{\beta} \omega^{2}+\varphi_{\alpha} \frac{d \omega}{d t} \\
\frac{d e_{\beta}}{d t} & =\varphi_{\alpha} \omega^{2}+\varphi_{\beta} \frac{d \omega}{d t} .
\end{aligned}
$$

From (5), the following EMF observer can be proposed,

$$
\begin{aligned}
& \frac{d \hat{e}_{\alpha}}{d t}=-\hat{\varphi}_{\beta} \hat{\omega}^{2}+\hat{\varphi}_{\alpha} \frac{d \hat{\omega}}{d t}+g\left(L \frac{d \hat{i}_{\alpha}}{d t}-L \frac{d i_{\alpha}}{d t}\right) \\
& \frac{d \hat{e}_{\beta}}{d t}=\hat{\varphi}_{\alpha} \hat{\omega}^{2}+\hat{\varphi}_{\beta} \frac{d \hat{\omega}}{d t}+g\left(L \frac{d \hat{i}_{\beta}}{d t}-L \frac{d i_{\beta}}{d t}\right),
\end{aligned}
$$

where $\hat{x}$ is the estimated value of $x$, and the time derivatives of stator currents are used as correction terms. An appropriate gain $g$ must be chosen when adjusting the observer in order to obtain desired convergence as shown in [29].

The estimated current derivatives, necessary for obtaining the correction term, can be calculated by (1),

$$
\begin{aligned}
& \frac{d \hat{i}_{\alpha}}{d t}=-\frac{R}{L} i_{\alpha}+\frac{1}{L} \hat{e}_{\alpha}-\frac{1}{L} v_{\alpha} \\
& \frac{d \hat{i}_{\beta}}{d t}=-\frac{R}{L} i_{\beta}+\frac{1}{L} \hat{e}_{\beta}-\frac{1}{L} v_{\beta} .
\end{aligned}
$$

Furthermore, the time derivative of the estimated speed used in (6) can be obtained from (2), assuming that driving torque is unknown,

$$
\hat{a}=\frac{d \hat{\omega}}{d t}=-\frac{1}{J}\left(\hat{\varphi}_{\alpha} i_{\alpha}+\hat{\varphi}_{\beta} i_{\beta}\right)-\frac{B}{J} \hat{\omega} .
$$

The estimate of the machine's speed can be found taking into account (3) and (4), so,

$$
e_{\alpha}^{2}+e_{\beta}^{2}=\omega^{2}\left(\varphi_{\alpha}^{2}+\varphi_{\beta}^{2}\right),
$$

and,

$$
\hat{\omega}=\sqrt{\frac{\hat{e}_{\alpha}^{2}+\hat{e}_{\beta}^{2}}{\hat{\varphi}_{\alpha}^{2}+\hat{\varphi}_{\beta}^{2}}} .
$$

The calculation of the measured current derivative, used in the correction term (6), may end up in a noisy estimation. To avoid that, the following change of variables is proposed,

$$
\begin{aligned}
& \hat{\zeta}_{\alpha}=\hat{e}_{\alpha}+g L i_{\alpha} \\
& \hat{\zeta}_{\beta}=\hat{e}_{\beta}+g L i_{\beta} .
\end{aligned}
$$


Taking time derivative of (11), and substituting from (6) and (7), the proposed observer will result in,

$$
\begin{aligned}
& \frac{d \hat{\zeta}_{\alpha}}{d t}=-\hat{\varphi}_{\beta} \hat{\omega}^{2}+\hat{\varphi}_{\alpha} \hat{a}+g\left(-R i_{\alpha}+\hat{e}_{\alpha}-v_{\alpha}\right) \\
& \frac{d \hat{\zeta}_{\beta}}{d t}=\hat{\varphi}_{\alpha} \hat{\omega}^{2}+\hat{\varphi}_{\beta} \hat{a}+g\left(-R i_{\beta}+\hat{e}_{\beta}-v_{\beta}\right),
\end{aligned}
$$

and the estimated EMF can be obtained by solving (11),

$$
\begin{aligned}
& \hat{e}_{\alpha}=\hat{\zeta}_{\alpha}-g L i_{\alpha} \\
& \hat{e}_{\beta}=\hat{\zeta}_{\beta}-g L i_{\beta} .
\end{aligned}
$$

The estimated position can be calculated from the estimated EMF as follows,

$$
\hat{\theta}=\tan ^{-1}\left(\frac{-\hat{e}_{\alpha}}{\hat{e}_{\beta}}\right),
$$

and the estimated position derivative of flux is calculated from (4) evaluated in the estimated position. The complete algorithm of the proposed observer can be found in the Appendix.

\section{Sampling of vibrations signals}

As shown in Figure 1, the rotor position estimated by the observer is used to obtain vibration measurement signals at equally-spaced rotor position instants. In this way, we obtain the same number of samples in a complete rotor turn, independently of the wind turbine speed. Moreover, since the proposed observer uses current and voltage measurements to estimate rotor position, sampling signal is independent of the load supplied by the generator. In this work, to obtain vibrations of constant position intervals, two different methods are proposed as described below.

1) Resampling at constant position intervals - If vibration signals are sampled at constant time intervals, it is possible to implement offline the proposed resampling at constant position intervals. As an example, Figure 2 shows the proposed method implemented for a number of samples $n=5$. Vector $\hat{\vec{\theta}}$ contains position data obtained from the EMF observer sampled at constant time intervals:

$$
\hat{\vec{\theta}}=\left[\begin{array}{llll}
\hat{\theta}_{(1)} & \hat{\theta}_{(2)} & \ldots & \hat{\theta}_{(n)}
\end{array}\right]
$$

These data are obtained at the same sampling instants than the vibration signal, as shown in the example of Figure 2 (a),

$$
\vec{x}=\left[\begin{array}{llll}
x_{(1)} & x_{(2)} & \ldots & x_{(n)}
\end{array}\right]
$$

For resampling vibration signal, vector of positions is redefined at constant intervals given by

$$
\begin{aligned}
{\hat{\overrightarrow{\theta^{*}}}}^{*} & =\left[\begin{array}{lllll}
\hat{\theta}_{(1)}^{*} & \hat{\theta}_{(2)}^{*} & \hat{\theta}_{(3)}^{*} & \ldots & \hat{\theta}_{(n)}^{*}
\end{array}\right] \\
& =\left[\begin{array}{lllll}
\hat{\theta}_{(1)} & \hat{\theta}_{(1)}+\Delta \theta & \hat{\theta}_{(1)}+2 \Delta \theta & \ldots & \hat{\theta}_{(n)}
\end{array}\right]
\end{aligned}
$$

where

$$
\Delta \theta=\hat{\theta}_{(k+1)}^{*}-\hat{\theta}_{(k)}^{*}=\frac{\hat{\theta}_{(n)}-\hat{\theta}_{(1)}}{n-1}
$$
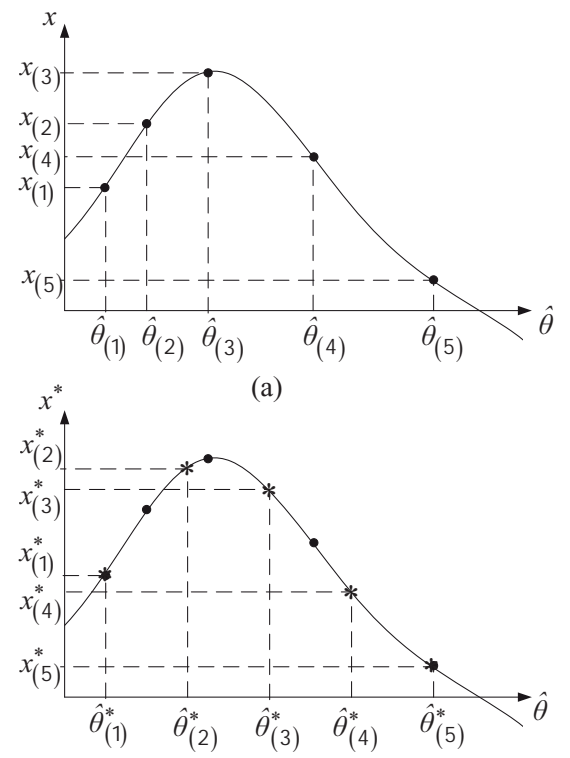

(b)

Fig. 2. Proposed resampling method: (a) data sampled at constant time intervals, (b) data resampled at constant position intervals.

Then, vibration signal resampled at constant position intervals are obtained by interpolation as shown in Figure 2 (b),

$$
\vec{x}^{*}=\left[\begin{array}{llll}
x_{(1)}^{*} & x_{(2)}^{*} & \ldots & x_{(n)}^{*}
\end{array}\right]
$$

Unlike [19], the proposed resampling method does not perform interpolation of the vector of acquisition times so that, for the same amount of data the number of interpolations is reduced by half.

2) Sampling at constant position intervals - If the strategy is implemented in a Digital Signal Processor (DSP) it is possible to sample vibration signals at intervals of constant position in real time, using the estimated position of the observer. In this way there is no need to perform the resampling as in the previous proposal. In Figure 3 a block diagram with the proposed strategy for sampling the vibration signals is shown. Both, phase currents and line voltages are sampled at constant time intervals. Then, the sampled signals are transformed to an $\alpha-\beta$ reference frame and used as inputs in the EMF observer. The EMF is used for position estimation as described in subsection II.B. Finally, the estimated position is used to determine the sampling instants for the vibration signals. The intervals between samples are chosen according to the number of samples per revolution $m$ that are necessary for the detection of faults.

\section{Fault detection strategy}

Vibration signals sampled at equally-spaced rotor positions allows to process the signals using the usual methods (i.e. frequency spectrum), avoiding the need to use some timefrequency analysis. Once obtained the frequency spectrum of vibration signals, clear spectrum components associated to each fault can be identified, simplifying diagnosis tasks. A scheme for the fault detection strategy is shown in Figure 4. Rotor imbalance produces an increase in the 


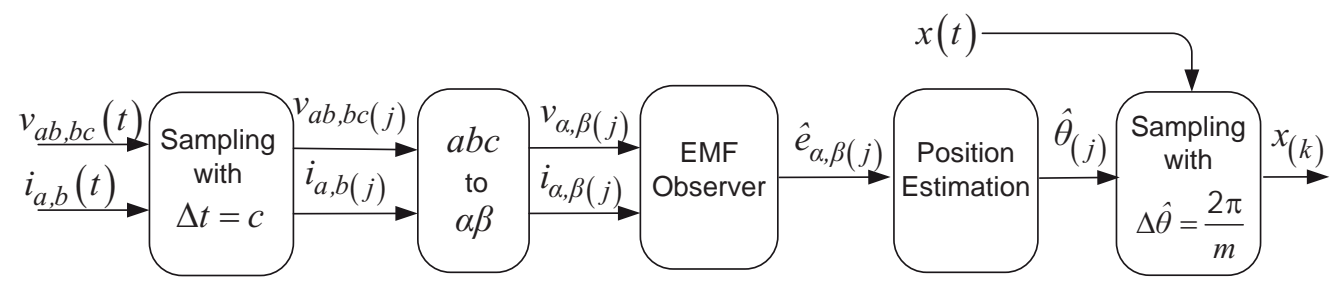

Fig. 3. Proposed strategy for sampling at constant position intervals.

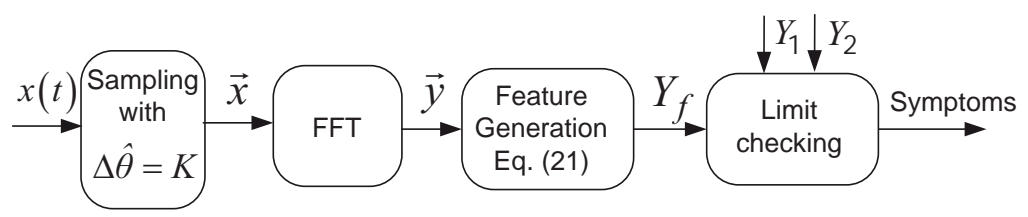

Fig. 4. Scheme of the proposed fault detection strategy.

vibration component at one cycle per rotor revolution. For a sampling at intervals $\Delta \theta$ and data set length of $n$, the resolution of the frequency spectrum in cycles per revolution $(\triangle C P R)$ is given by,

$$
\triangle C P R=\frac{2 \pi}{n \Delta \theta}
$$

Then, if the vector $\vec{y}=\left[\begin{array}{llll}y_{(1)} & y_{(2)} & \ldots & y_{(n)}\end{array}\right]$ corresponds to the absolute values of the frequency spectrum components, the component at one cycle per revolution is $y_{(k)}$ with $k=$ $\frac{1}{\triangle C P R}=\frac{n \Delta \theta}{2 \pi}$. Then, the component used for detection of the imbalance $Y_{f}$ is extracted form the vector $\vec{y}$ as follows,

$$
Y_{f}=\max \left(\left[\begin{array}{lll}
y_{(k-b)} & \cdots & y_{(k+b)}
\end{array}\right]\right)
$$

where $b$ defines a narrow band around the $k$ component.

As a first approach, in this paper a limit checking of absolutes values is proposed. Thresholds are selected based on experience and initial measurement on the system. As used in most applications of fault diagnosis with vibration monitoring, two limits are defined, one for triggering an alarm $Y_{1}$ and a second limit $Y_{2}$ to immediately stop the turbine (fault). Constant alarm limits may result conservatives to detect faults at low speed, due to the level of vibrations produced by imbalance usually increases with speed. This is not a significant problem because the effects of imbalance are more harmful when the turbine speed increase. As future work, it is of interest to develop an adaptive threshold to enhance the sensitivity of the detection strategy. Moreover, with the objective of extracting the component $Y_{f}$ another computational techniques such as correlation [30] can be used. However, the use of the FFT also allows to obtain other components in order to extend the strategy for detecting other types of faults (e.g. bearings, gear or generator).

In the following section experimental validation of the proposal is presented.

\section{EXPERIMENTAL RESULTS}

Figure 5 shows the test bench used for obtaining the experimental results. It is composed by the PMSG under test,

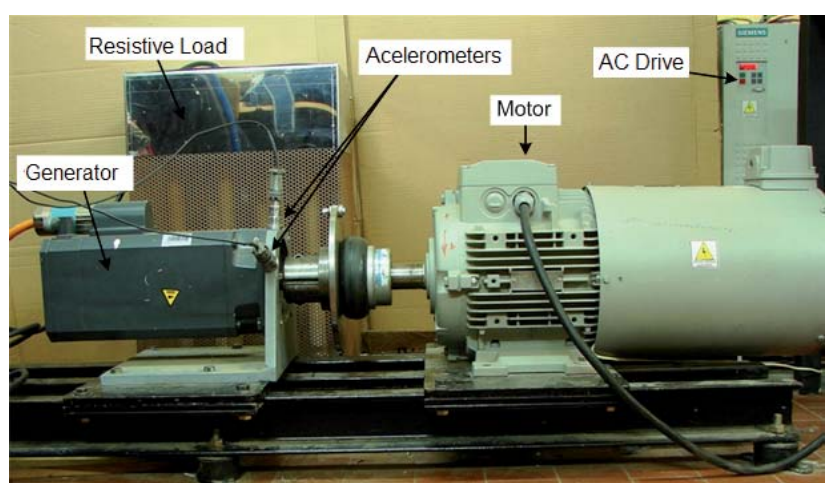

Fig. 5. Test bench.

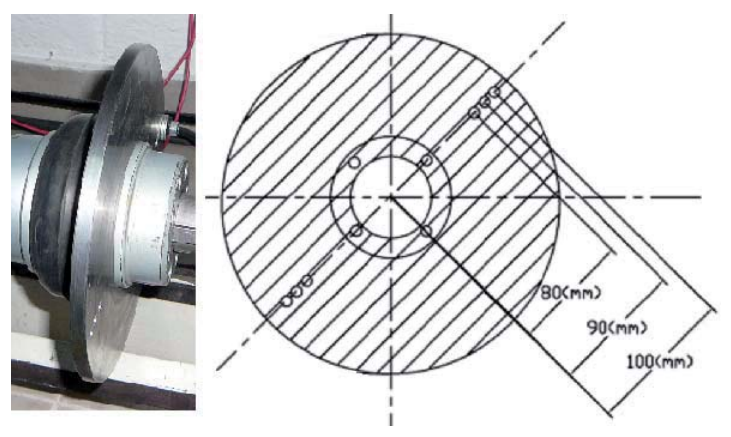

Fig. 6. Special plate placed between the PMSG and the induction motor drive.

which is driven by a variable speed induction motor drive. PMSG parameters are shown in Table I. A special plate is coupled between the PMSG and the induction motor drive, which allows including a mass at different distances from the center of rotation, thus producing different rotor imbalance levels (see Figure 6).

PMSG vibration is measured through accelerometers mounted on the generator frame; and velocity vibration values were obtained according to ISO 10816 [31]. Two output currents are measured through AC current clamps, while 


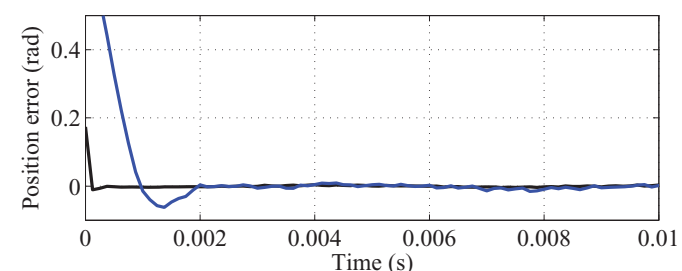

(a)

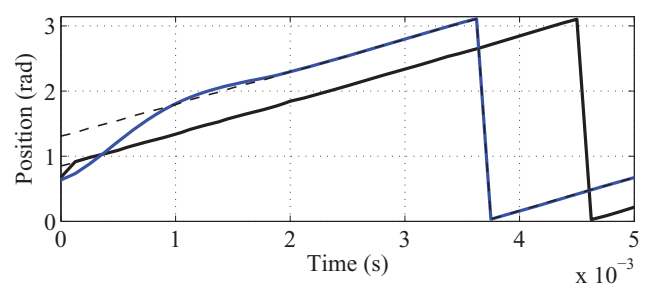

(b)

Fig. 7. Observer convergence for $g=2000$ (black line) and $g=400$ (blue line). (a) Position estimation error and (b) Actual (dashed line) and estimated (solid line) position.

two stator voltages are measured using $200 \mathrm{MHz}$ bandwidth isolated test probes. All measurements are acquired using an oscillographic register and later processed in Matlab using the proposed resampling at constant position intervals method. In the implementation of the technique 64000 samples of vibrations and electric variables (currents and voltages) were acquired at $8 \mathrm{KS} / \mathrm{s}$ sampling frequency.

Observer gain was first designed following the procedure proposed in [29], and later adjusted taking into account that the selection of $g$ results from a trade-off between observer convergence speed and measurement noise influence. Thus, a $g=2000$ value was selected for all the experimental results. Estimation position convergence is shown in Figure 7, for two different values of gain $g$. Figure 7 (a) shows the estimation error for $g=2000$ (black) and $g=400$ (blue), in order to show that the proposed observer is stable for different gain values. Actual and estimated position for these two gain values are presented in Figure 7 (b). The observer was implemented in discrete time, with a sampling period of $125 \mu \mathrm{s}$, that is the same sampling frequency of the acquired signals.

Different tests were performed to validate the proposed strategy. These tests include constant speed and generator load, variable speed operation of the loaded generator, and variable speed operation with a sudden load change. All the cases were tested with and without rotor imbalance.

\section{A. Constant speed and load test}

The first tests were performed at constant speed operation, with a constant three-phase resistance connected at the generator terminals. Figure 8 shows the velocity vibration spectrum for the PMSG without rotor imbalance (a), and with rotor imbalance (b). As it can be appreciated, a clear component at one time rotation speed appear in the spectrum, which increase its amplitude when rotor imbalance increases. In this case, since rotor speed is constant, there is no need of resampling, since vibration components can be clearly
TABLE I

PMSG data and parameters

\begin{tabular}{cc}
\hline Rated torque & $16,6 \mathrm{Nm}$ \\
\hline Rated current & $7,7 \mathrm{~A}$ \\
\hline Rated power & $3,5 \mathrm{~kW}$ \\
\hline Rated speed & $2000 \mathrm{rpm}$ \\
\hline Inertia & $0,0046 \mathrm{~kg} \cdot \mathrm{m}^{2}$ \\
\hline Poles & 8 \\
\hline$R$ & $1,396 \Omega$ \\
\hline$L$ & $9,354 \mathrm{mH}$ \\
\hline$\Phi$ & $0,297 \mathrm{~Wb} / \mathrm{m}^{2}$ \\
\hline
\end{tabular}

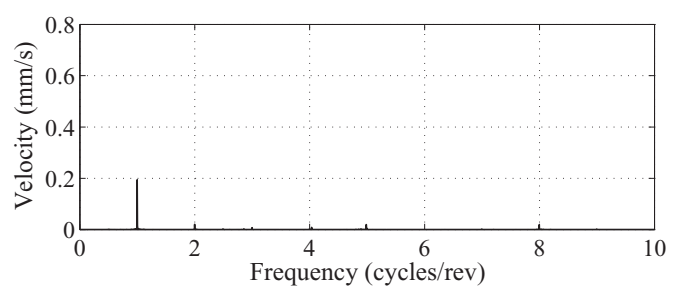

(a)

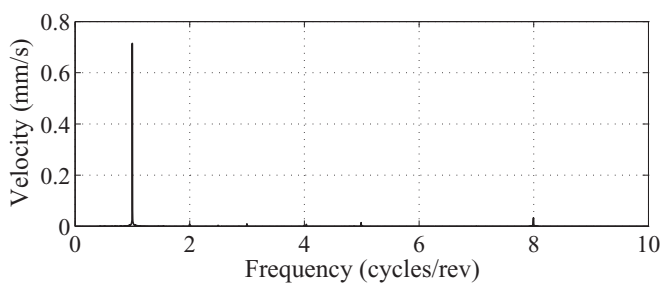

(b)

Fig. 8. Velocity vibrations at constant speed and load. (a) Balanced and (b) with imbalance.

identified in the spectrum.

\section{$B$. Variable speed with a constant load resistance}

In these tests, PMSG is operated at variable speed, while the constant resistance is connected at its terminals. Figure 9 (a) shows velocity vibrations measured at the PMSG frame, 9 (b) and 9 (c) shows the EMF and rotor speed estimated by the proposed observer, for the balanced rotor case. Variable speed operation makes that frequency components at the vibration spectrum cannot be clearly appreciated if constant sampling time is used, as can be appreciated in Figure 10 (a). However, when vibration signals are resampled synchronized with the estimated rotor position, velocity vibration spectrum shows clear components, as it can be seen in Figure 10 (b).

A similar effect can be appreciated on the spectrum of the generator voltage, Figure 11. As it can be appreciated, once resampled using the proposed strategy, the component at four times (the electric frequency) is clearly defined in the spectrum of Figure 11 (b). So, this technique can be also used for detecting electrical faults.

The case with rotor imbalance is presented in Figures 12 and 13. As it can be appreciated in Figure 12, vibration level grows with unbalance, as expected. However, from the vibration spectrum with the original sampling (Figure 13 (a)) this growth cannot be appreciated nor quantified (compare with Figure 10 (a)). Once resampled using the proposed 


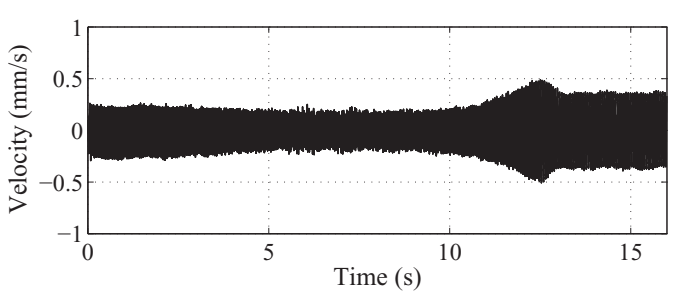

(a)

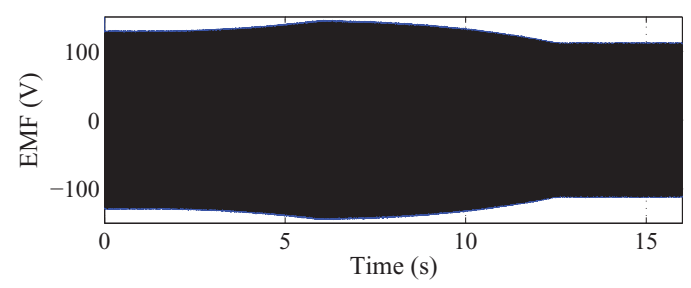

(b)

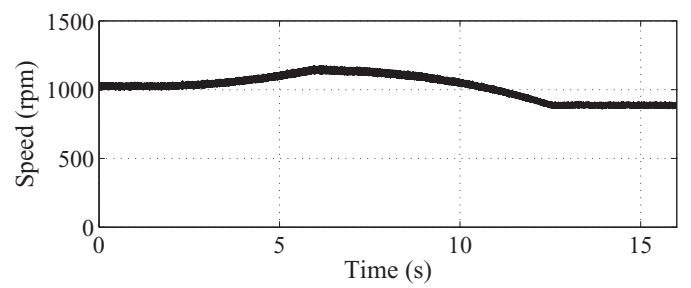

(c)

Fig. 9. Balanced machine at variable speed supplying a constant load. (a) Velocity vibrations, (b) estimated EMF and (c) estimated rotor speed.

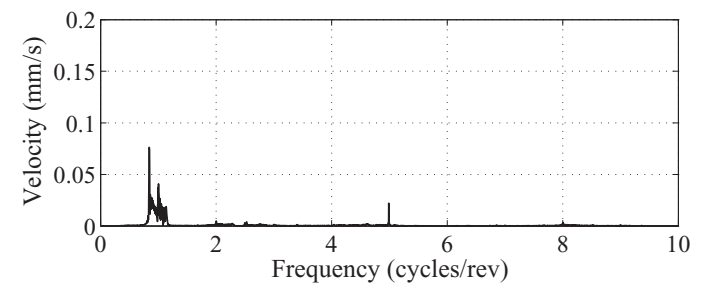

(a)

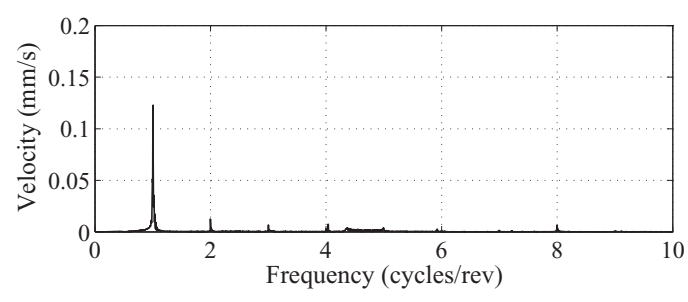

(b)

Fig. 10. Velocity vibrations for a balanced machine at variable speed supplying a constant load. (a) Original sample and (b) resampled.

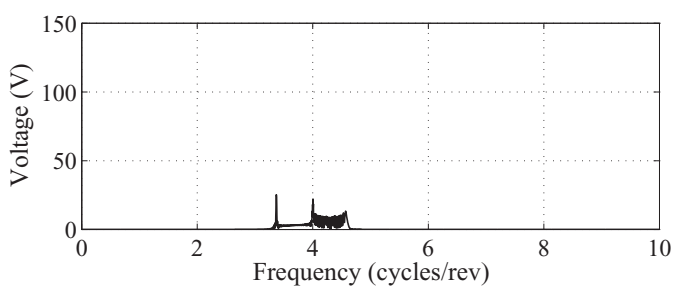

(a)

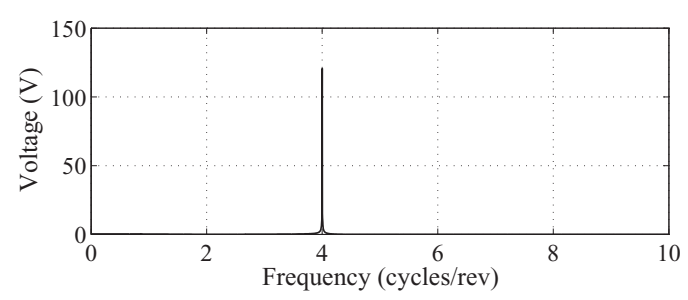

(b)

Fig. 11. Voltage spectrum for a balanced machine at variable speed supplying a constant load. (a) Original sample and (b) resampled.

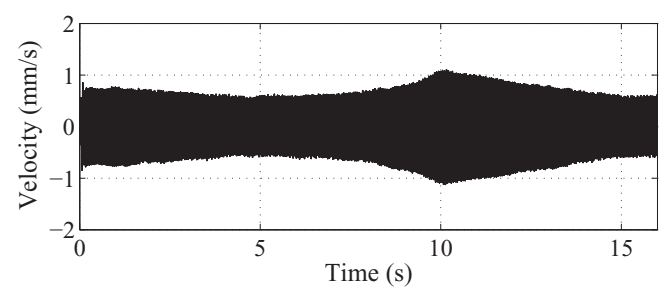

(a)

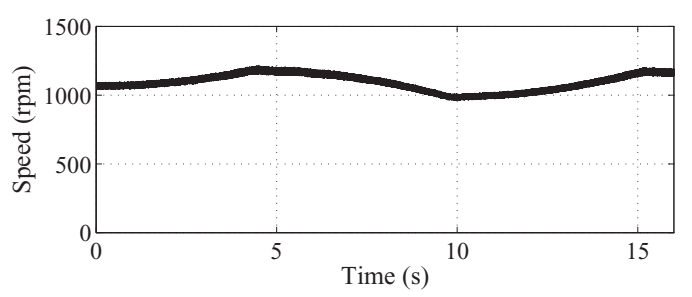

(b)

Fig. 12. Unbalanced machine at variable speed supplying a constant load. (a) Velocity vibrations, and (b) estimated rotor speed.

technique, the growth of the one time component due to imbalance can be clearly appreciated (Figure 13 (b)).

\section{Behavior under load changes}

These tests were performed in order to show the robustness of the proposed strategy against sudden load changes. The PMSG is operating at constant speed at no load, when a resistive load is connected at generator terminals during the measurement interval. Figure 14 shows the machine velocity vibrations and the velocity vibration spectrum for the balanced case, while Figure 15 corresponds to the unbalanced machine test. As it can be appreciated in Figure 14 (a) and Figure 15 (a), load connection produces a transient in machine vibrations. However, the spectra obtained using the proposed resampling method allows to clearly appreciate the magnitude of the one 


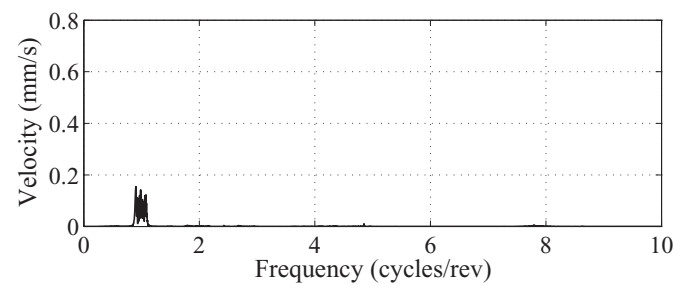

(a)

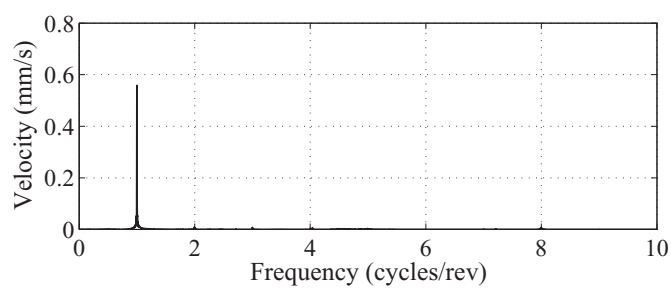

(b)

Fig. 13. Velocity vibrations for a unbalanced machine at variable speed supplying a constant load. (a) Original sample and (b) resampled.

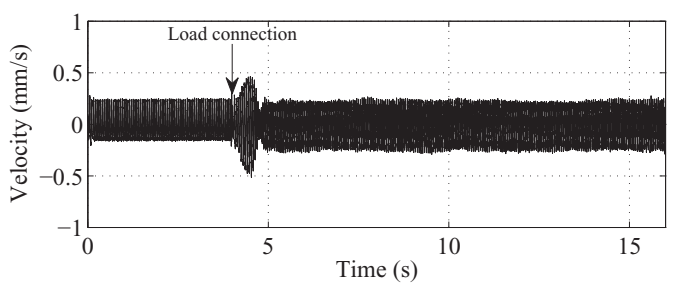

(a)

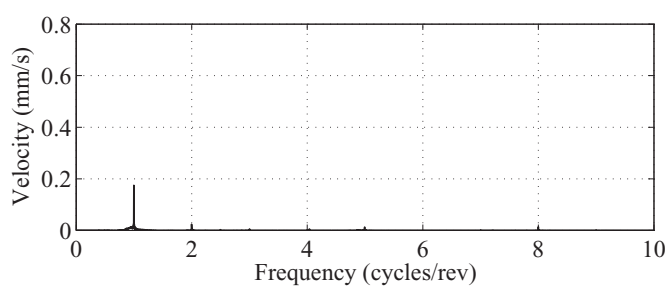

(b)

Fig. 14. Balanced machine at constant speed during load connection. (a) Velocity vibrations, and (b) Velocity vibration spectrum (resampled using the proposed observer).

time component, as well as distinguish the balanced and the unbalanced case, as can be seen in Figures 14 (b) and 15 (b). These spectra can be compared with those obtained in the constant speed and load test (Figure 8).

In the next tests, the PMSG is first operating at variable speed at no load, and a resistive load is suddenly connected at generator terminals during the measurement interval. The balanced rotor test is presented in Figures 16 and 17. Vibration velocity signal and the estimated rotor speed are shown in Figures 16 (a) and 16 (b), respectively. As it can be seen in Figure 17 (b), the most clear component in the vibration spectrum is the one at one time. However, this component cannot be clearly appreciated using the original sampling

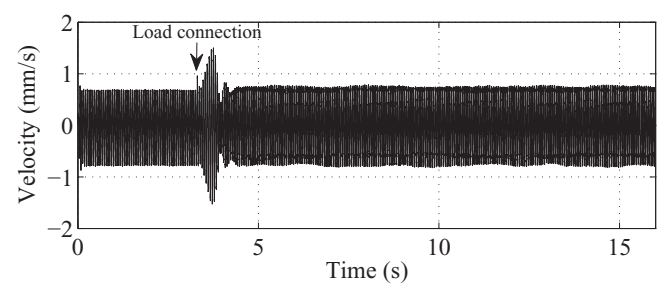

(a)

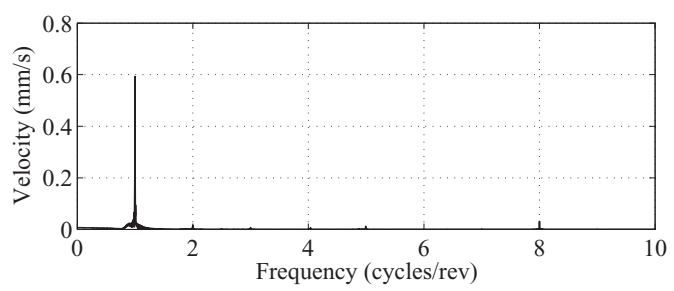

(b)

Fig. 15. Unbalanced machine at constant speed during load connection. (a) Velocity vibrations, and (b) Velocity vibration spectrum (resampled using the proposed observer).

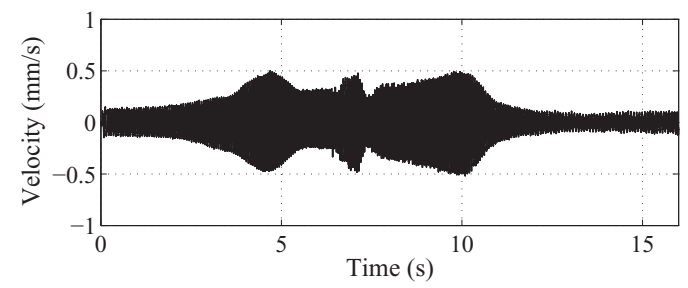

(a)

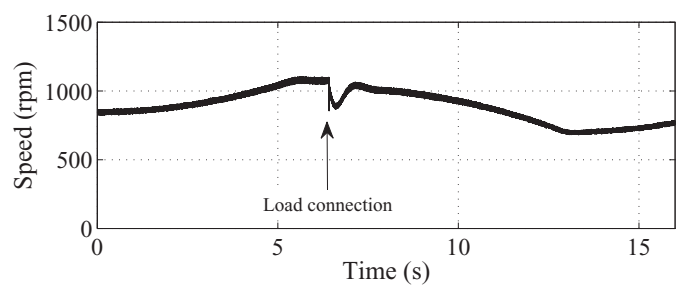

(b)

Fig. 16. Balanced machine at variable speed during load connection. (a) Velocity vibrations, and (b) estimated rotor speed.

(Figure 17 (a)).

Figures 18 and 19 shows the results obtained for rotor imbalance. Again, vibration signal of Figure 18 (a) shows a growth due to imbalance, when compared with Figure 16 (a). However, the magnitude of such growth can only be appreciated in the spectrum of the resampled vibration signal, shown in Figure 19 (b), where the clear one time component is seen.

\section{Comparison with voltage based resample}

In order to show the need of using the proposed observer, Figure 20 (a) shows the vibration spectrum when vibration signal is resampled using the angle of stator voltages. Even when this resampling technique helps improving vibration 


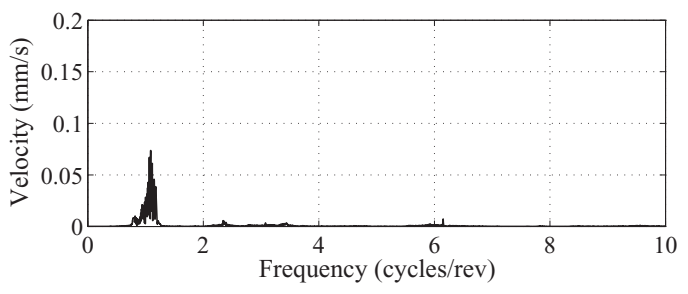

(a)

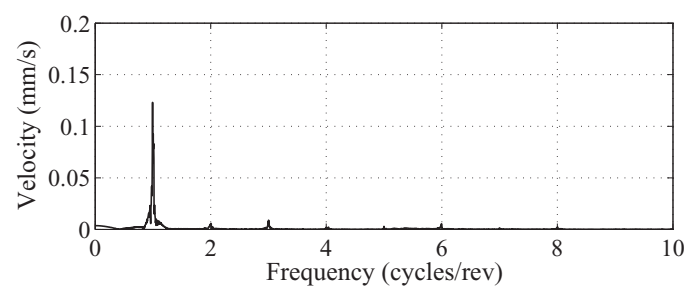

(b)

Fig. 17. Velocity vibrations for a balanced machine at variable speed during load connection. (a) Original sample and (b) resampled.

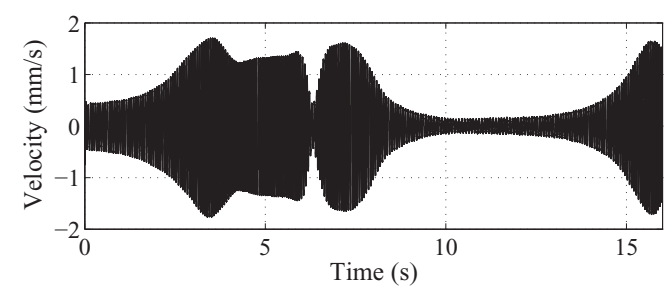

(a)

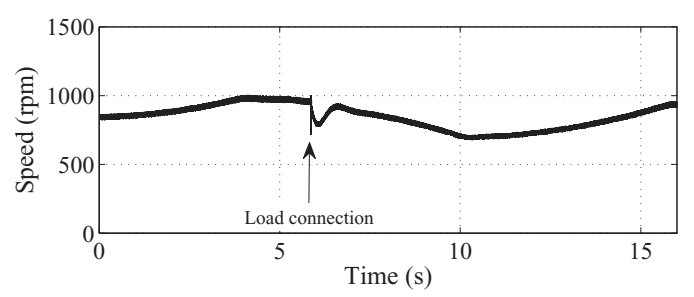

(b)

Fig. 18. Unbalanced machine at variable speed during load connection. (a) Velocity vibrations and (b) estimated rotor speed.

spectrum, this angle is affected by load changes. On the contrary, the proposed observer based technique is not affected by load changes, which produces a more clear vibration spectrum, as shown in Figure 20 (b). This fact can be better explained with the help of Figure 21. In Figure 21 (a), module of the measured voltage (calculated from its $\alpha-\beta$ components) and of the estimated EMF are compared. As it can be appreciated, once the load is connected, stator voltage amplitude (dashed line) changes due to the voltage drop produced in the generator impedance. On the other hand, estimated EMF amplitude (solid line) is only affected by the speed change, but not due to load change. A similar effect is produced in the angle of the measured voltage. Figure 21 (b) shows the difference between the angle of stator voltage and the vector of equally spaced angles generated

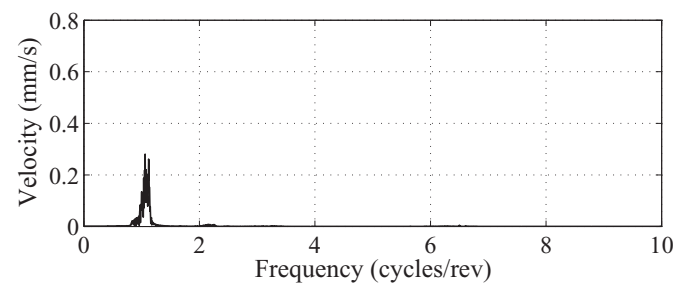

(a)

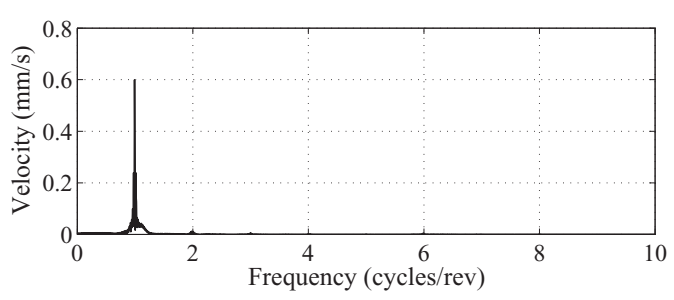

(b)

Fig. 19. Velocity vibrations for an unbalanced machine at variable speed during load connection. (a) Original sample, and (b) resampled using the proposed observer.

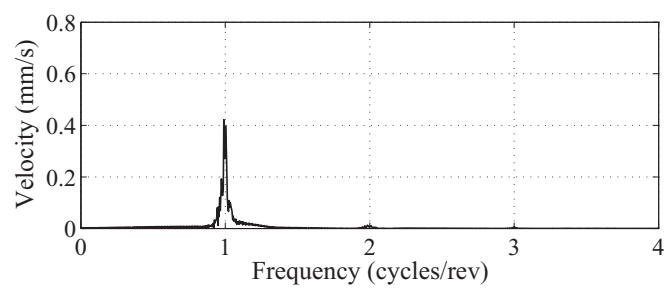

(a)

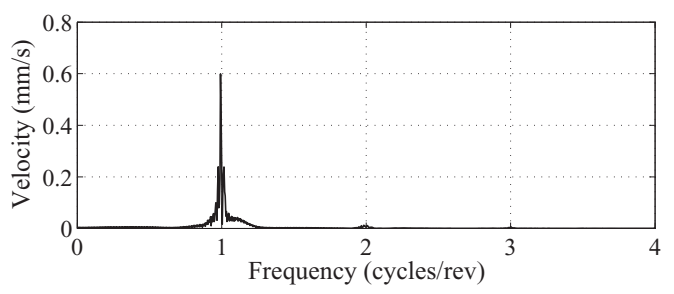

(b)

Fig. 20. Velocity vibrations for an unbalanced machine during load connection. (a) Resampled using voltages and (b) resampled using the proposed observer.

for resampling (dashed line), and the difference between the estimated position and the vector of equally spaced angles (solid line). As it can be seen, before the load is connected to the PMSG, both angles coincides, and the variation is only produced by speed changes. However, once the load is connected, these angles are different, due to the effect of the generator impedance on the stator voltages. Thus, the estimated position using the proposed observer allows a robust resampling, almost independent of generator load changes.

\section{E. Sensitivity to parameter variations}

Since the proposed observer is based on the PMSG model, sensitivity of the proposed strategy to variation or errors 


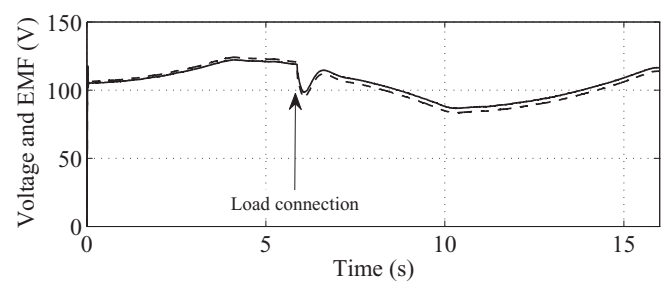

(a)

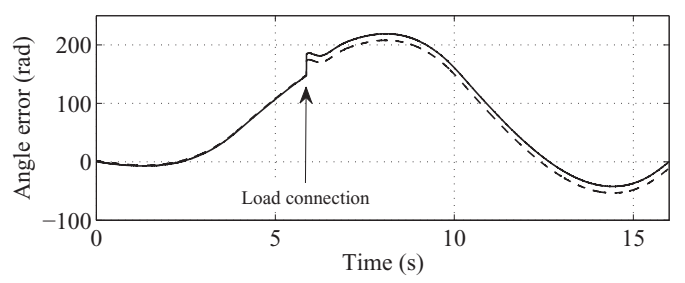

(b)

Fig. 21. Module (a) and angle difference (b) of the measured voltages (dashed line) and estimated EMF (solid line).

in the model parameters is evaluated in this section. The proposed strategy was evaluated using the estimated positions obtained from the observer with the rated parameters and three additional cases: with half the inductance value $0.5 \mathrm{~L}$, with half the resistance value $0.5 R$ and with double resistance value $2 R$. Then, the estimated position was used for implementing the off-line resampling method for the same case presented in the previous section.

As it can be appreciated in Figure 22 (a), parameter errors introduce an error in the estimated EMF amplitude, when compared to the EMF estimated using the rated parameters (dashed line). However, as shown in Figure 22 (b) and 22 (c), the error in the estimated position with respect to the obtained using the rated parameters is very low (below $0.01 \mathrm{rad})$. Therefore, the influence of parameter errors in the final result, which is the resampled vibration spectrum, is negligible, as can be seen in Figure 22 (d). In this figure, the spectra obtained for the rated parameters case and the other three cases are superimosed. As it can be appreciated, there is not difference between them.

\section{CONCLUSIONS}

In this work, a new method for processing vibration signals was proposed in order to detect and diagnose faults in variable speed wind turbines. The proposed technique is based on a resampling of the acquired vibration signals in order to obtain a speed independent spectrum. A reduced order observer was designed for estimating rotor position using generator voltage and current measurements, without the need of a position sensor. Thus, the estimated position was used for resampling vibration signals. A disadvantage of our approach over other approaches, such as the one presented in [19], is its higher computational cost due to the implementation of the observer used for position estimation. Experimental results for different generator operating conditions were presented. It was demonstrated that the proposed strategy allows obtaining

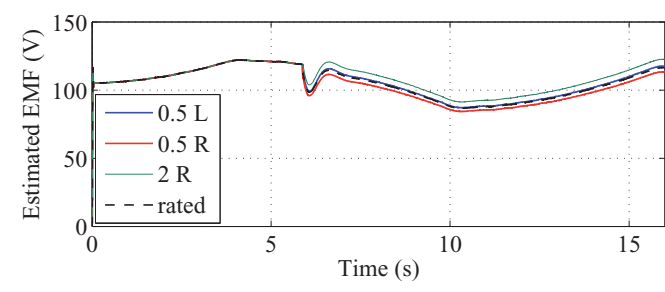

(a)

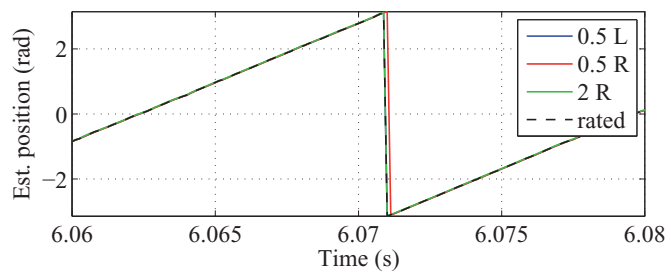

(b)

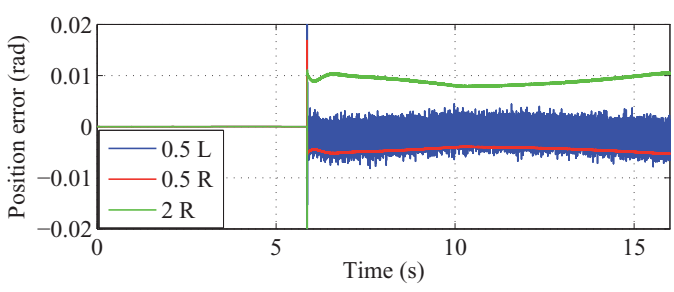

(c)

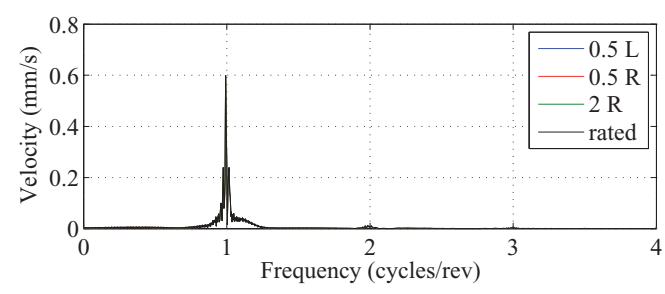

(d)

Fig. 22. Sensitivity to parameter variation. (a) Module of the estimated EMF, (b) estimated position, (c) difference with the estimated position using the rated parameters, (d) velocity vibration spectrum.

well defined frequency components in the vibration spectrum even under variable speed and load conditions.

\section{APPENDIX}

Considering the previously stated equations, the observer algorithm can be implemented as follows,

- Initial conditions: $\hat{\theta}(0), \hat{\omega}(0), \hat{e}_{\alpha}(0), \hat{e}_{\beta}(0)$

- Inputs: $i_{\alpha}, i_{\beta}, v_{\alpha}, v_{\beta}$

- Algorithm:

$$
\begin{gathered}
\hat{\varphi}_{\alpha}=-\Phi \sin (\hat{\theta}) \\
\hat{\varphi}_{\beta}=\Phi \cos (\hat{\theta}) ; \\
\hat{a}=\frac{1}{J}\left(\hat{\varphi}_{\alpha} i_{\alpha}+\hat{\varphi}_{\beta} i_{\beta}\right)-\frac{B}{J} \hat{\omega} ;
\end{gathered}
$$




$$
\begin{array}{r}
\frac{d \zeta_{\alpha}}{d t}=-\hat{\varphi}_{\beta} \hat{\omega}^{2}+\hat{\varphi}_{\alpha} \hat{a}+g\left(-R i_{\alpha}-\hat{e}_{\alpha}+v_{\alpha}\right) \\
\frac{d \zeta_{\beta}}{d t}=\hat{\varphi}_{\alpha} \hat{\omega}^{2}+\hat{\varphi}_{\beta} \hat{a}+g\left(-R i_{\beta}-\hat{e}_{\beta}+v_{\beta}\right) ; \\
\hat{e}_{\alpha}=\zeta_{\alpha}-g L i_{\alpha} \\
\hat{e}_{\beta}=\zeta_{\beta}-g L i_{\beta} ; \\
\hat{\theta}=\operatorname{atan} 2\left(-\hat{e}_{\alpha}, \hat{e}_{\beta}\right) ; \\
\hat{\omega}=\operatorname{sign}\left(\frac{\hat{\theta}_{(k)}-\hat{\theta}_{(k-1)}}{T_{s}}\right) \sqrt{\frac{\hat{e}_{\alpha}^{2}+\hat{e}_{\beta}^{2}}{\hat{\varphi}_{\alpha}^{2}+\hat{\varphi}_{\beta}^{2}}} \\
\text { ACKNOWLEDGEMENT }
\end{array}
$$

\section{ACKNOWLEDGEMENT}

This work was supported by Consejo Nacional de Investigaciones Científicas y Técnicas, Argentina; Universidad Nacional de Río Cuarto, Argentina, and Ministerio de Ciencia, Tecnología e Innovación Productiva, Argentina.

\section{REFERENCES}

[1] T. Ackermann, Ed., Wind power in power systems. Chichester, UK: John Wiley \& Sons, Ltd, 2005.

[2] B. Lu, Y. Li, X. Wu, and Z. Yang, "A review of recent advances in wind turbine condition monitoring and fault diagnosis," in IEEE Power Electronics and Machines in Wind Applications (PEMWA 2009), 2009, pp. 1-7.

[3] B. C. P. Lau, E. W. M. Ma, and M. Pecht, "Review of offshore wind turbine failures and fault prognostic methods," in IEEE Conference on Prognostics and System Health Management (PHM), 2012, pp. 1-5.

[4] N. Freire, J. Estima, and A. Marques Cardoso, "Open-circuit fault diagnosis in pmsg drives for wind turbine applications," IEEE Transactions on Industrial Electronics, vol. 60, no. 9, pp. 3957-3967, Sept 2013.

[5] B. Tang, T. Song, F. Li, and L. Deng, "Fault diagnosis for a wind turbine transmission system based on manifold learning and shannon wavelet support vector machine," Renewable Energy, vol. 62, pp. 1-9, 2014.

[6] J. Sheldon, G. Mott, H. Lee, and M. Watson, "Robust wind turbine gearbox fault detection," Wind Energy, vol. 17, no. 5, pp. 745-755, 2014.

[7] L. Wenyi, W. Zhenfeng, H. Jiguang, and W. Guangfeng, "Wind turbine fault diagnosis method based on diagonal spectrum and clustering binary tree svm," Renewable Energy, vol. 50, pp. 1-6, 2013.

[8] Z. Feng and M. Liang, "Fault diagnosis of wind turbine planetary gearbox under nonstationary conditions via adaptive optimal kernel timefrequency analysis," Renewable Energy, vol. 66, pp. 468 - 477, 2014.

[9] W. Yang, P. J. Tavner, C. J. Crabtree, and M. Wilkinson, "Cost-effective condition monitoring for wind turbines," IEEE Transactions on Industrial Electronics, vol. 57, no. 1, pp. 263-271, 2010.

[10] M. Blodt, J. Regnier, and J. Faucher, "Distinguishing load torque oscillations and eccentricity faults in induction motors using stator current wigner distributions," IEEE Transactions on Industry Applications, vol. 45, no. 6, pp. 1991-2000, 2009.
[11] Z. Zhang, A. Verma, and A. Kusiak, "Fault analysis and condition monitoring of the wind turbine gearbox," IEEE Transactions on Energy Conversion, vol. 27, no. 2, pp. 526-535, 2012.

[12] X. Gong and W. Qiao, "Imbalance fault detection of direct-drive wind turbines using generator current signals," IEEE Transactions on Energy Conversion, vol. 27, no. 2, pp. 468-476, 2012.

[13] S. Djurovic, C. J. Crabtree, P. J. Tavner, and A. C. Smith, "Condition monitoring of wind turbine induction generators with rotor electrical asymmetry," IET Renewable Power Generation, vol. 6, no. 4, pp. 207216, 2012.

[14] M. Fernández-Cabanas and M. García-Melero, Técnicas para el mantenimiento diagnóstico de máquinas eléctricas rotativas. Barcelona: Marcombo, S.A., 1998.

[15] K. C. Kim, S. B. Lim, K. B. Jang, S. G. Lee, J. Lee, Y. G. Son, Y. K. Yeo, and S. H. Baek, "Analysis on the direct-driven high power permanent magnet generator for wind turbine," in Eighth International Conference on Electrical Machines and Systems (ICEMS 2005), vol. 1, 2005, pp. 243-247.

[16] J. N. Stander, M. J. Kamper, and G. Venter, "Analytic modelling and optimization of slip synchronous permanent magnet wind turbine generator topologies," Wind Energy, p. n/a, 2014.

[17] Z. Hameed, Y. S. Hong, Y. M. Cho, S. H. Ahn, and C. K. Song, "Condition monitoring and fault detection of wind turbines and related algorithms: A review," Renewable and Sustainable Energy Reviews, vol. 13, no. 1, pp. 139, 2009.

[18] F. P. García Márquez, A. M. Tobias, J. M. Pinar Pérez, and M. Papaelias, "Condition monitoring of wind turbines: Techniques and methods," Renewable Energy, vol. 46, pp. 169-178, 2012.

[19] L. F. Villa, A. Reñones, J. R. Perán, and L. J. de Miguel, "Angular resampling for vibration analysis in wind turbines under non-linear speed fluctuation," Mechanical Systems and Signal Processing, vol. 25, no. 6, pp. 21572168, 2011.

[20] R. Krishnan, Electric motor drives: modeling, analysis, and control. Prentice Hall PTR, 2001.

[21] C. H. De Angelo, "Control para máquinas de ca de imanes permanentes con fem arbitraria, sin sensores mecánicos," Ph.D. dissertation, Universidad Nacional de La Plata, 2004.

[22] C. De Angelo, G. Bossio, J. Solsona, G. O. Garcia, and M. I. Valla, "A rotor position and speed observer for permanent-magnet motors with nonsinusoidal emf waveform," IEEE Transactions on Industrial Electronics, vol. 52, no. 3, pp. 807-813, 2005.

[23] F. Briz and M. W. Degner, "Rotor position estimation," IEEE Industrial Electronics Magazine, vol. 5, no. 2, pp. 24-36, June 2011.

[24] B. Fischer, "Reducing rotor speed variations of floating wind turbines by compensation of non-minimum phase zeros," IET Renewable Power Generation, vol. 7, no. 4, pp. 413-419, July 2013. 
[25] F. D. Bianchi, H. De Battista, and R. J. Mantz, Wind turbine control systems: principles, modelling and gain scheduling design. Springer, 2006.

[26] L. Trilla, F. D. Bianchi, and O. Gomis-Bellmunt, "Linear parameter-varying control of permanent magnet synchronous generators for wind power systems," IET Power Electronics, vol. 7, no. 3, pp. 692-704, March 2014.

[27] M. Stiebler, Wind energy systems for electric power generation. Springer, 2008.

[28] M. Chinchilla, S. Arnaltes, and J. C. Burgos, "Control of permanent-magnet generators applied to variablespeed wind-energy systems connected to the grid," IEEE Transactions on Energy Conversion, vol. 21, no. 1, pp. 130-135, March 2006.

[29] J. Solsona, M. I. Valla, and C. Muravchik, "A nonlinear reduced order observer for permanent magnet synchronous motors," IEEE Transactions on Industrial Electronics, vol. 43, no. 4, pp. 492-497, 1996.

[30] R. Isermann, Fault-Diagnosis Systems: An Introduction from Fault Detection to Fault Tolerance. Germany: Springer, 2006.

[31] ISO 10816-1:1995, Mechanical vibration - Evaluation of machine vibration by measurements on non-rotating parts. Geneva, Switzerland: International Organization for Standardization, 1995.

\section{BIOGRAPHIES}

José Maria Bossio was born in Italó, Argentina, in 1979. He received the Electrical Engineer degree from the Universidad Nacional de Río Cuarto, Río Cuarto, Argentina, in 2006, the MsC. of Engineering Sciences degree from the Universidad Nacional de Río Cuarto, Río Cuarto, Argentina, in 2010, and the Dr. of Control Systems degree from the Universidad Nacional del Sur, Bahía Blanca, Argentina, in 2012.

Since 2001, he has been with the Grupo de Electrónica Aplicada, Facultad de Ingeniería, Universidad Nacional de Río Cuarto. He is currently Teaching Assistant at Universidad Nacional de Río Cuarto and Assistant Researcher at Consejo
Nacional de Investigaciones Científicas y Técnicas, Argentina. Dr. Bossio is Member of the IEEE. His research interests include fault diagnosis on electric machines, and renewableenergy generation.

Guillermo Rubén Bossio was born in Italó, Argentina, in 1974. He received the Electrical Engineer degree from the Universidad Nacional de Río Cuarto, Río Cuarto, Argentina, in 1999 and the Dr. of Engineering degree from the Universidad Nacional de La Plata, La Plata, Argentina, in 2004.

Since 1994, he has been with the Grupo de Electrónica Aplicada, Facultad de Ingeniería, Universidad Nacional de Río Cuarto. He is currently Associate Professor at Universidad Nacional de Río Cuarto and Independt Researcher at Consejo Nacional de Investigaciones Científicas y Técnicas, Argentina. His research interests include fault diagnosis on electric machines, electric vehicles, and renewable-energy generation.

Dr. Bossio is Member of the IEEE. He has been a member of the organizing committees of several national conferences. $\mathrm{He}$ is also member of the Automatic Control Association of Argentina.

Cristian Hernán De Angelo was born in Río Cuarto, Argentina, in 1974. He received the Electrical Engineer degree from the Universidad Nacional de Río Cuarto, Río Cuarto, Argentina, in 1999 and the Dr. of Engineering degree from the Universidad Nacional de La Plata, La Plata, Argentina, in 2004.

Since 1994, he has been with the Grupo de Electrónica Aplicada, Facultad de Ingeniería, Universidad Nacional de Río Cuarto. He is currently Associate Professor at Universidad Nacional de Río Cuarto and Independt Researcher at Consejo Nacional de Investigaciones Científicas y Técnicas, Argentina. His research interests include fault diagnosis on electric machines, electric vehicles, and renewable-energy generation.

Dr. De Angelo is Senior Member of the IEEE. He has been member of the organizing committees of several national conferences. He is also member of the Automatic Control Association of Argentina. 